\title{
Electrons on a spherical surface: Physical properties and hollow spherical clusters
}

\author{
Dario Cricchio, Emilio Fiordilino, and Franco Persico \\ Dipartimento di Fisica, Università di Palermo, Via Archirafi 36, 90123 Palermo, Italy
}

(Received 13 March 2012; published 11 July 2012)

\begin{abstract}
We discuss the physical properties of a noninteracting electron gas constrained to a spherical surface. In particular we consider its chemical potentials, its ionization potential, and its electric static polarizability. All these properties are discussed analytically as functions of the number $N$ of electrons. The trends obtained with increasing $N$ are compared with those of the corresponding properties experimentally measured or theoretically evaluated for quasispherical hollow atomic and molecular clusters. Most of the properties investigated display similar trends, characterized by a prominence of shell effects. This leads to the definition of a scale-invariant distribution of magic numbers which follows a power law with critical exponent -0.5 . We conclude that our completely mechanistic and analytically tractable model can be useful for the analysis of self-assembling complex systems.
\end{abstract}

DOI: 10.1103/PhysRevA.86.013201

PACS number(s): 61.48.-c, 73.22.-f, 89.75.Da

\section{INTRODUCTION}

The fabrication of quasi-two-dimensional materials has stimulated theoretical research on a mathematical model describing an electron gas in two dimensions, which has been dubbed 2D jellium. This model is constituted by a number $N$ of electrons constrained on a planar surface. Moreover, in order to guarantee its overall electrical neutrality, the planar surface is assumed to be rigid and uniformly positively charged. Such a model has recently been adopted to discuss the properties of high-density 2D jellium [1,2]. In this paper we wish to extend the $2 \mathrm{D}$ jellium model to cover the case of $N$ electrons on a positively charged spherical surface and to discuss its basic physical properties. At this stage we shall neglect for simplicity all electron-electron interactions. A similar spherical model (with reference to $\pi$ electrons) has been proposed in the past to explain the properties of magnetic shielding in neutral fullerenes [3] and also in fullerene polarizability calculations [4] and in the magnetic properties of capped nanoparticles of nonmagnetic substances [5]. In addition theoretical models of electron gas on a spherical surface have recently been discussed with particular reference to the form of the exchange and correlation energy [6,7].

Mathematically, the problem of quantizing the dynamics of an electron constrained to a curved surface $S$ is related to that of embedding $m$-dimensional manifolds in $n$-dimensional Euclidean spaces $(n>m)$ [8-10]. The main features of the theory can be summarized as follows.

(1) A system of curvilinear coordinates $q_{1}, q_{2}$ is established on the curved surface, and a strong potential $\lambda V\left(q_{3}\right)$ is introduced in order to confine the electron to the immediate neighborhood of $S ; \lambda$ defines the strength of the potential.

(2) The wave function of the electron is factorized in a normal part $\psi_{n}\left(q_{3}\right)$ and in a tangential part $\psi_{t}\left(q_{1}, q_{2}\right)$.

(3) The Schrödinger equation is separated into an equation for $\psi_{n}\left(q_{3}\right)$ and one for $\psi_{t}\left(q_{1}, q_{2}\right)$. The eigenvalue spectrum of the $\psi_{n}\left(q_{3}\right)$ part is taken as nondegenerate, and only its lowest eigenvalue is populated.

(4) The strong limit $\lambda \rightarrow \infty$ for the potential is taken.

*emilio.fiordilino@unipa.it
(5) The Schrödinger equation for $\psi_{t}\left(q_{1}, q_{2}\right)$ then takes the form

$$
i \hbar \frac{\partial \psi_{t}}{\partial t}=-\frac{\hbar^{2}}{2 m} \nabla^{2} \psi_{t}-V_{s} \psi_{t}
$$

where $\nabla^{2}$ is the Laplacian in curvilinear coordinates $\left(q_{1}, q_{2}\right)$ and $V_{s}$ is an effective potential,

$$
V_{s}\left(q_{1}, q_{2}\right)=-\frac{\hbar^{2}}{8 m}\left(k_{1}^{2}-k_{2}^{2}\right) \text {. }
$$

In this expression $k_{1}$ and $k_{2}$ are the principal curvatures of $S$ at each point $\left(q_{1}, q_{2}\right)$ on the surface. We note that $V_{s}$ vanishes for a spherical surface. Moreover gauge fields do not appear in the equation for $\psi_{t}\left(q_{1}, q_{2}\right)$ due to the assumption of a nondegenerate spectrum for $\psi_{n}\left(q_{3}\right)[11,12]$.

This paper is organized as follows. In Sec. II we present the main results of the theory of an electron gas constrained on a spherical surface, and we define its chemical potentials along with other physically relevant quantities, such as its ionization energy and its static polarizability. In Sec. III we briefly review some of the physical properties of hollow quasispherical clusters of atoms which have been measured experimentally or theoretically predicted by others using relatively sophisticated models. In Sec. IV we compare the results of our model with those reviewed is Sec. III. In Sec. V we present the conclusions which we have been able to draw from the analysis of our model.

\section{ELECTRON GAS ON A SPHERICAL SURFACE}

The allowed energy levels for an electron constrained to a spherical surface of radius $R$ are

$$
E_{\ell}=\frac{\hbar^{2}}{2 m_{e} R^{2}} \ell(\ell+1),
$$

where $m_{e}$ is the mass of the electron and $\ell$ is 0 or a positive integer and each energy level (or shell) is $(2 \ell+1)$-fold degenerate, corresponding to the $2 \ell+1$ possible values of $m(-\ell-1 \leqslant m \leqslant \ell+1)$.

We now proceed to fill the lowest-lying levels of the spherical shell with the $N$ available electrons, taking into account that a level with a given $\ell$ can accommodate $2(2 \ell+1)$ 
electrons. Suppose that the last completely full shell has $\ell=L$. Then the number $N_{L}$ of electrons with $\ell \leqslant L$ is

$$
N_{L}=2 \sum_{\ell=0}^{L}(2 \ell+1)=2(L+1)^{2},
$$

where the factor 2 is motivated by Pauli's principle requirement; in what follows we shall dub the numbers $N_{L}$ given by Eq. (4) as magic numbers, and we shall qualify as noble the configuration of the $2 \mathrm{D}$ jellium spherical particle. The total energy of a noble configuration is thus

$$
\begin{aligned}
W_{L} & =\frac{\hbar^{2}}{2 m_{e} R^{2}} 2 \sum_{\ell=0}^{L} \ell(\ell+1)(2 \ell+1) \\
& =\frac{\hbar^{2}}{2 m_{e} R^{2}} L(L+1)\left(L^{2}+3 L+2\right),
\end{aligned}
$$

and its Fermi energy $E_{F}$ is

$$
E_{F}=\frac{\hbar^{2}}{2 m_{e} R^{2}} L(L+1) .
$$

On the other hand, it is generally possible that some of the $N$ electrons must be accommodated in a shell (with $\ell=L+1$ ) beyond the last completely full shell. Suppose their number is $n_{L+1}$. Then the total energy of any of the lowest-energy configurations of the $N$ electrons is, in general,

$$
\begin{aligned}
W_{N}= & \frac{\hbar^{2}}{2 m_{e} R^{2}}\left[L(L+1)\left(L^{2}+3 L+2\right)\right. \\
& \left.+n_{L+1}(L+1)(L+2)\right],
\end{aligned}
$$

and its Fermi energy, the energy required to add one electron, is

$$
E_{F}=\frac{\hbar^{2}}{2 m_{e} R^{2}}\left[\left(1-\delta_{n_{L+1}, 0}\right)(L+1)(L+2)+\delta_{n_{L+1}, 0} L(L+1)\right] .
$$

Thus $E_{F}$, as a function of $N$, presents discontinuities at filledshell configurations (i.e., when $n_{L+1}$ vanishes).

As is well known [13], $E_{F}$ is the chemical potential of the electron gas, which is defined as $\partial W_{N} / \partial N=W_{N}-W_{N-1}$ since $N$ is a discrete variable. It can be checked that this is indeed the case using the expressions above. It should also be noted that $W_{N}-W_{N-1}$ is also a contribution to the ionization potential, the other contribution being the work against the Coulomb force necessary to bring one electron from the spherical surface to infinity.

We shall now consider more closely the background positive surface charge. Since the negative electrons are discrete, it is convenient, for accounting purposes, to discretize also the positive charge, which we shall indicate here by the term nuclear charge, whose value is $Z e$, with $e$ being the proton charge. In order to achieve overall electric neutrality, the number of nuclear charges $Z$ must be equal to that of the $N$ electrons in the system. We shall now assume that in our system the number of nuclear charges is proportional to the surface of the spherical shell, so that $\alpha Z=R^{2}$, where $\alpha=(4 \pi \sigma)^{-1}$, $\sigma$ being the nuclear number density on the spherical surface, which we also assume to be independent of $R$; in order to emphasize the role the nuclear charge, we shall introduce

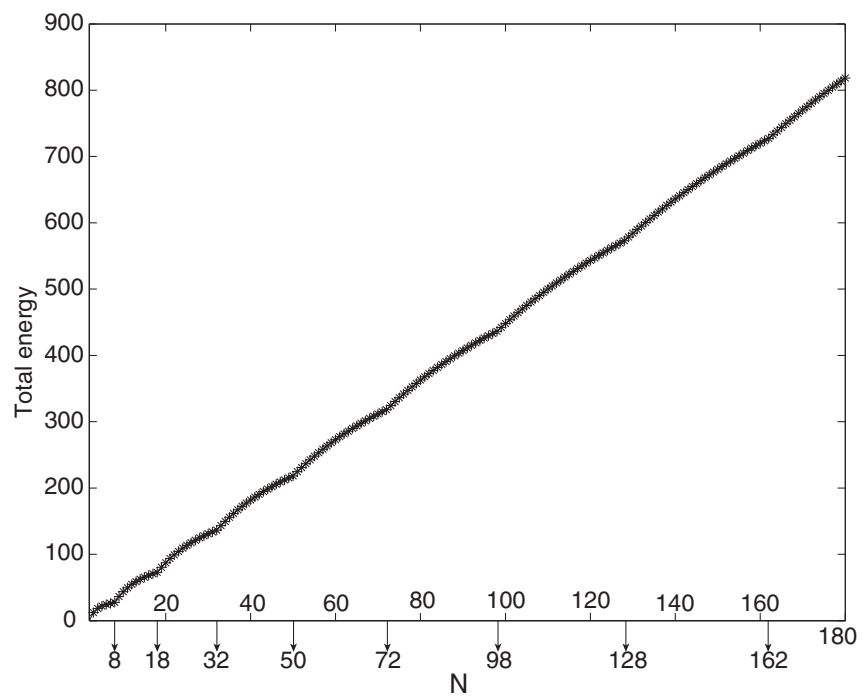

FIG. 1. Energy $W_{N, N}$ in eV of the electron gas vs the number $N$ of electrons. The arrows mark the position of the magic numbers (see text).

a second index in the total energy, which hereafter will be denoted by $W_{Z ; N}$. Thus, in the case of neutral 2D jellium system, we have

$$
\begin{aligned}
W_{N ; N}= & \frac{\hbar^{2}}{2 m_{e} \alpha N}\left[L(L+1)\left(L^{2}+3 L+2\right)\right. \\
& \left.+n_{L+1}(L+1)(L+2)\right]
\end{aligned}
$$

and

$$
\begin{aligned}
E_{F}= & \frac{\hbar^{2}}{2 m_{e} \alpha N}\left[\left(1-\delta_{n_{L+1}, 0}\right)(L+1)(L+2)\right. \\
& \left.+\delta_{n_{L+1}, 0} L(L+1)\right] .
\end{aligned}
$$

For later use we shall need the quantity $W_{N ; N-1}$; it is

$$
W_{N ; N-1}=W_{N ; N}-E_{F} .
$$

$W_{N ; N}$ and $E_{F}$ are plotted in Figs. 1 and 2 as functions of $N$; in all plots of this paper the magic numbers (full shells) are flagged by arrows on the horizontal axis. From Fig. 2 we clearly see that magic numbers correspond to the minima of the chemical potential and thus to stable configurations.

We emphasize that $W_{N ; N}$ in Eq. (9) is a function of $N$ different from $W_{N}$ in (7) due to the elimination of $R$ in favor of $N$. As a consequence, also

$$
\mu(N) \equiv \partial W_{N ; N} / \partial N=W_{N ; N}-W_{N-1 ; N-1}
$$

is a function of $N$ different from $E_{F}$ and corresponds to another chemical potential. In fact $\mu(N)$ is the change in the energy of the system due to the addition of an electron and of a nuclear charge; thus we realize that its minima correspond to stable configurations of the system. The quantities $\mu(N)$ and $\partial \mu(N) / \partial N$ are plotted numerically in Fig. 3, where the peaks in $\partial \mu(N) / \partial N$ indicate clearly the stable (closed-shell) configurations of spherical $2 \mathrm{D}$ jellium for magic numbers. Joining the peaks of $\partial \mu(N) / \partial N$ by straight segments yields the polygonal shown in Fig. 4.

At this stage of the discussion it is straightforward to calculate the ionization potential of the surface; actually, in 


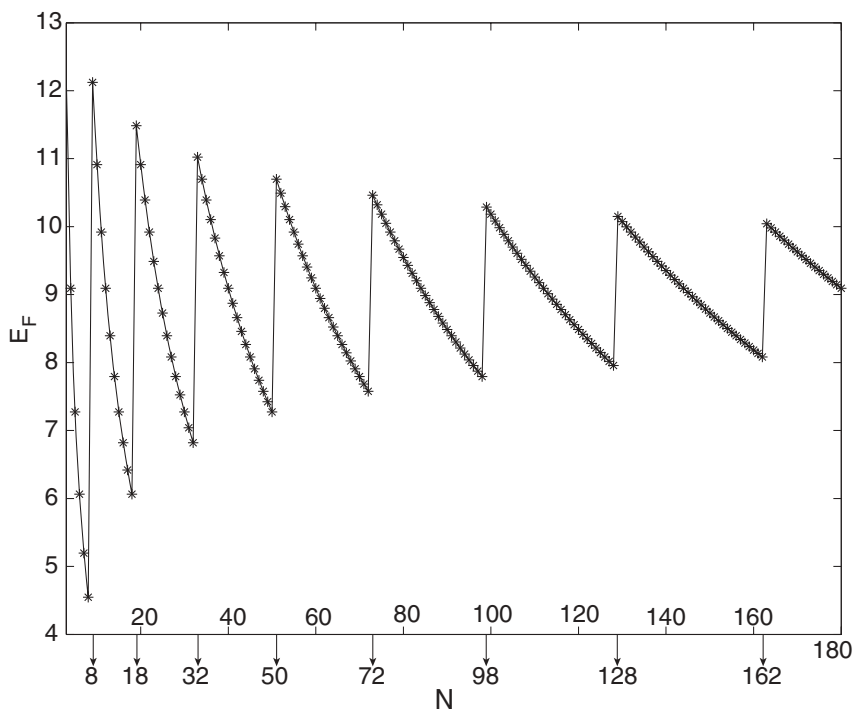

FIG. 2. The Fermi energy $E_{F}(N)$ from Eq. (10) of the system in $\mathrm{eV}$ vs the number $N$ of electrons. $E_{F}(N)$ is the energy of the uppermost electron in the neutral system. The solid line is an interpolation.

order to remove one electron from the $2 \mathrm{D}$ jellium surface to infinity an energy is needed, which is the sum

$$
I_{p}(N)=\left(e^{2} / R\right)+W_{N ; N-1}-W_{N ; N} .
$$

Here we are assuming that the positive charge left behind on the sphere is uniformly distributed over the surface of radius $R$. The quantity $I_{p}(N)$ is plotted numerically in Fig. 5.

The model accounts also for the electric static polarizability; in fact in the presence of a static electric field $\mathcal{E}$ directed along the $z$ axis of the polar coordinate system, the Hamiltonian

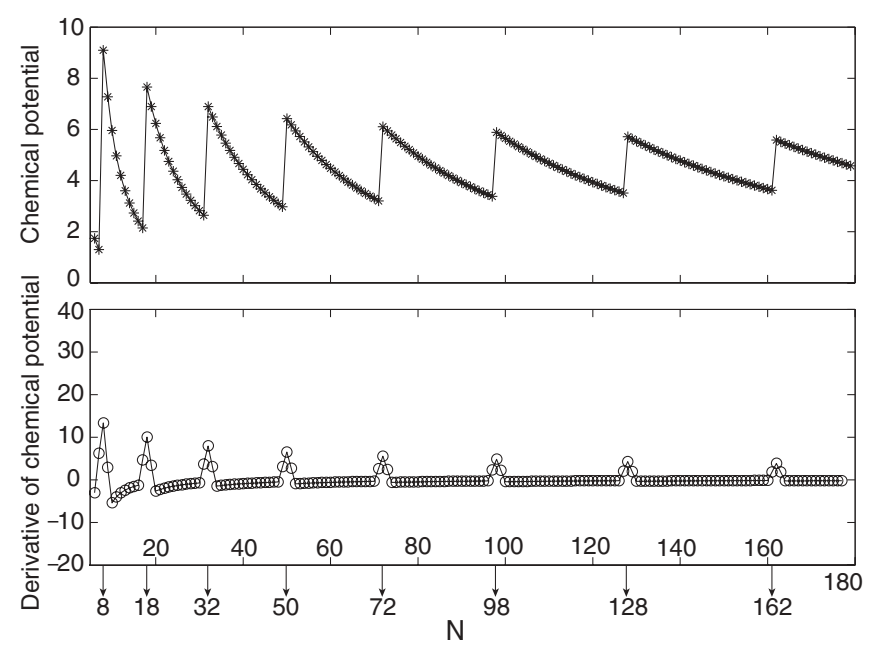

FIG. 3. The chemical potential $\mu(N) \equiv W_{N, N}-W_{N-1, N-1}$ of the system (stars) in eV vs the number $N$ of electrons (the solid line is an interpolation); $\mu(N)$ is the change in energy of the system for when one nuclear and one electron charge are added to the system, and it is the first derivative of the total energy plotted in Fig. 1. Circles are the second derivative of $W_{N, N}$ in $\mathrm{eV}$.

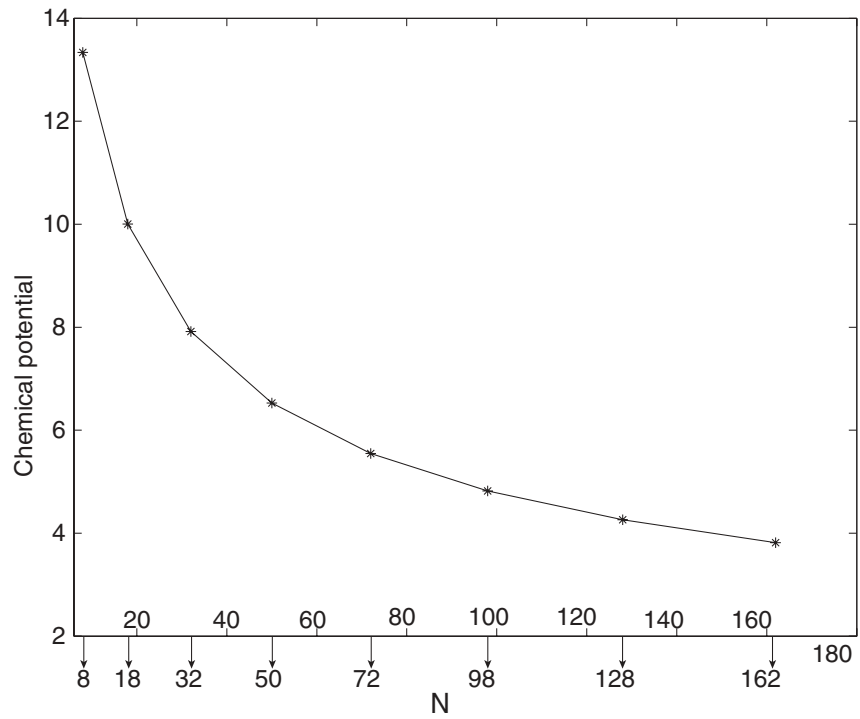

FIG. 4. The stability polygonal in $\mathrm{eV}$; it is obtained by joining the peaks of the bottom plot of Fig. 3.

is

$$
\hat{H}=\frac{\hbar^{2}}{2 m_{e} R^{2}} \hat{L}^{2}+\hbar \Omega_{0} \cos \theta,
$$

where $\Omega_{0}=e \mathcal{E} R / \hbar$ and $\hat{L}^{2}|\ell, m\rangle=\ell(\ell+1)|\ell, m\rangle$.

Second-order perturbation theory yields a shift with respect to the unperturbed energy levels in Eq. (3) given by

$$
\begin{gathered}
\Delta E_{\ell, m}=m_{e} R^{2} \Omega_{0}^{2} \frac{\ell(\ell+1)-3 m^{2}}{\ell(2 \ell-1)(\ell+1)(2 \ell+3)} \quad(\ell>0, \forall m), \\
\Delta E_{0,0}=-\frac{m_{e} R^{2} \Omega_{0}^{2}}{3} .
\end{gathered}
$$

Thus each shell is split into $2 \ell+1$ levels, $2 \ell$ of which (those with $m \neq 0$ ) are doublets. The electric polarizability of the

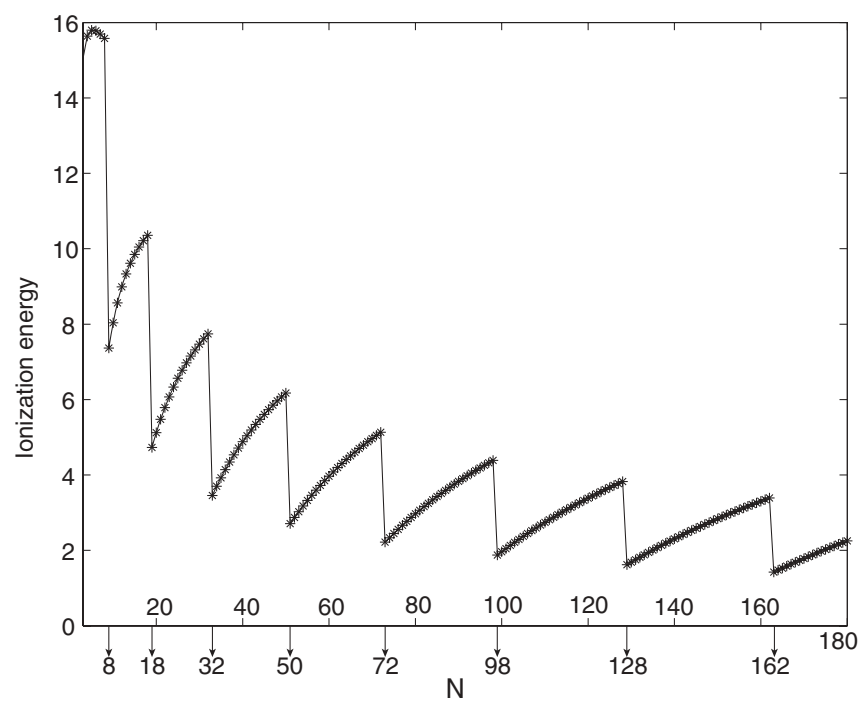

FIG. 5. The ionization energy $I_{p}$ of the system in $\mathrm{eV}$ vs the number $N$ of atoms; $I_{p}$ is the energy required to extract one electron from the surface. 
$|\ell, m\rangle$ state $\alpha_{\ell m}$ is obtained from (15) and (16) and from

$$
\Delta E_{\ell, m}=-\frac{\alpha_{\ell m}}{\mathcal{E}^{2}}
$$

Consider now a completely full shell with $0<\ell \leqslant L$ and $(2 \ell+1) m$ sublevels, each containing two electrons. Then the total polarizability of the shell is $\alpha_{\ell}=\sum_{m} 2 \alpha_{\ell m}$ for $\ell \neq 0$ and

$$
\alpha_{\ell}=\frac{m_{e} R^{4}}{\hbar^{2}} \frac{e^{2} \ell^{2}}{2 \ell(\ell+1)-3 / 2} \quad(0<\ell \leqslant L),
$$

where we have used $\sum_{m} m^{2}=\ell(\ell+1)(2 \ell+1)$. Expression (18) is convenient to evaluate numerically the core polarizability $\alpha_{\text {core }}=\sum_{\ell} \alpha_{\ell}+\alpha_{\ell=0}$ of the system. The total polarizability is evaluated as $\alpha_{\text {core }}+\alpha_{\text {valence, }}$, where $\alpha_{\text {valence }}$ is the contribution of the $n_{L+1}$ electrons residing in the incomplete shell $\ell=L+1$. From (15)

$$
\begin{aligned}
\alpha_{\text {valence }}= & \frac{m_{e} R^{4}}{\hbar^{2}} e^{2} \sum_{m} f(m) \\
& \times \frac{(L+1)(L+2)-3 m^{2}}{(L+1)(2 L+1)(L+2)(2 L+5)},
\end{aligned}
$$

where $f(m)$ is the occupancy of the $m$ state within the valence shell. This indicates that the total polarizability of spherical 2D jellium as a function of $N$ is slightly different from the $N^{2}$ behavior of $\alpha_{\text {core }}$ due to the contribution of the $n_{L+1}$ electrons residing in the incomplete shell $\ell=L+1$. The contributions $\alpha_{\text {core }}$ and $\alpha_{\text {valence }}$ as functions of $N$ are evaluated numerically and are represented in Figs. 6 and 7 along with the total polarizability in Fig. 8. We have chosen to fill the $2 L+3$ states of the valence shell proceeding from $|m|=L+1$ to $m=0$ and thereafter repeating the procedure up to the exhaustion of the $n_{L+1}$ available electrons. It is worth noting that relevant points of the three polarizabilities can be interpolated by parabolas.

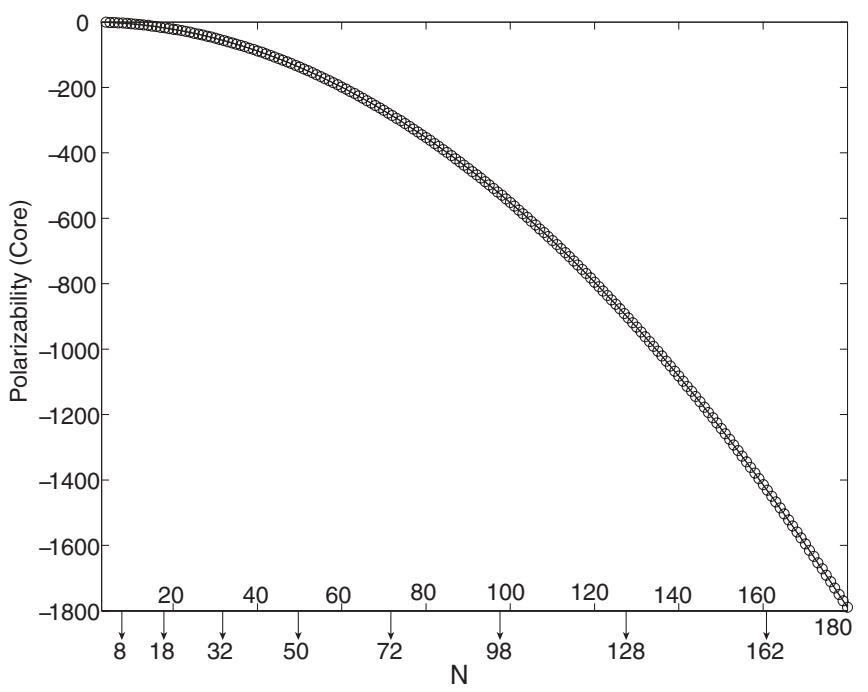

FIG. 6. Polarizability of the core electrons of the 2D jellium in $\AA^{3}$ vs the number $N$ of atoms. The polarizability can be interpolated by the parabola $\alpha_{\text {core }}=-0.055 N^{2}$.

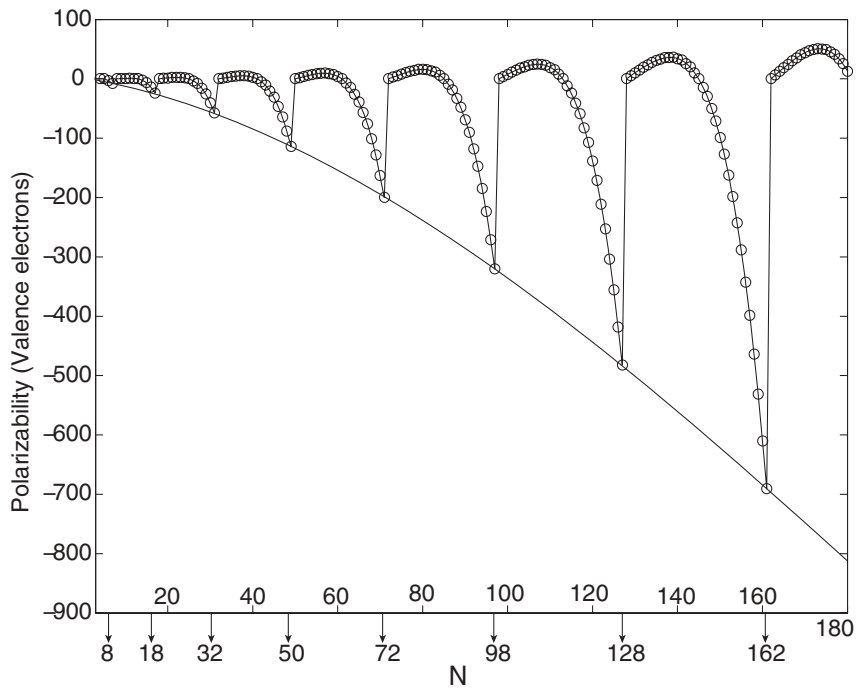

FIG. 7. Polarizability of the valence electrons of the $2 \mathrm{D}$ jellium in $\AA^{3}$ vs the number $N$ of atoms. The interpolating parabola $\alpha_{v}=$ $-0.026 N^{2}$ is shown.

\section{PHYSICAL PROPERTIES OF HOLLOW QUASISPHERICAL MOLECULES AND CLUSTERS OF ATOMS}

In this part we shall review briefly a selection of experimental and theoretical results on the physical properties of hollow quasispherical aggregates of atoms which have been published in recent years. The aim is to provide a basis for comparison of these properties with those obtained in the first part of the present work for the 2D jellium spherical model. Clearly, completeness of such a review is out of the question, given the vastness of the literature published on the subject of spherical hollow clusters.

We begin our analysis by examining the stability of the aggregates. Hollow clusters of noble metals present enhanced stability at some magic numbers of atoms in the cluster. For $\mathrm{Au}$ these magic numbers are $8,18,20$, and $34[14,15]$ as

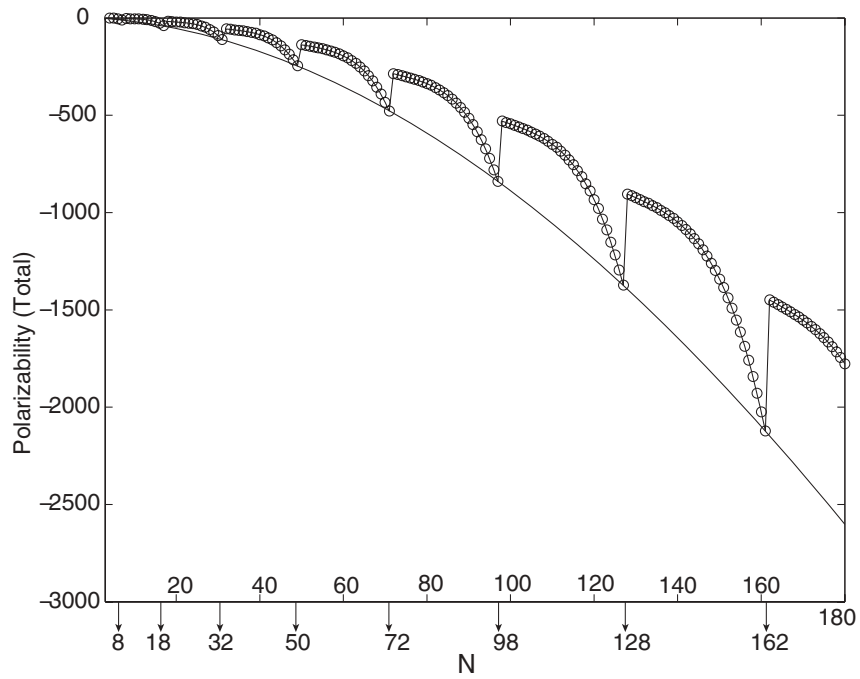

FIG. 8. Total polarizability of the $2 \mathrm{D}$ jellium in $\AA^{3}$ vs the number $N$ of atoms. The interpolating parabola $\alpha_{t}=-0.081 N^{2}$ is shown. 
well as 32 and $90[16,17]$ and 50 [18]. Also the much more popular hollow structures of the fullerene series present an abundance of magic numbers, such as $24,28,32,36,50,60$, and 70 [19-22] for small fullerenes $C_{n}(n<100)$ and 122, 130, 162,180 , and higher for large fullerenes $(n>100)$ [23,24]. A complete list of stable fullerenes is made impossible by the existence of a diverging number of isomers for each $n$. Nevertheless, Fig. 1 of [25] summarizes the binding energy of the fullerene series as a function of $\mathrm{C}$ atoms. A genetic algorithm has also been implemented to explore the stability of $(\mathrm{MgO})_{n}$ clusters, including a class of hollow quasispherical clusters, with magic numbers from 10 to 35 [26].

We examine now the ionization energy; this has been measured and calculated (using density-functional theory) [27] for a large series of the fullerenes from $\mathrm{C}_{20}$ to $\mathrm{C}_{180}$. As a function of the number of $\mathrm{C}$ atoms in the molecule, the points fall on a smooth curve, gently decreasing toward $7 \mathrm{eV}$, with clear superimposed discontinuous deviations of a few percent $[28,29]$.

As for static polarizability, a very popular family of hollow quasispherical molecules for which trends of the static electric polarizability have been calculated theoretically is that of the icosahedral fullerenes from $\mathrm{C}_{60}$ to $\mathrm{C}_{2160}$ [30] using selfconsistent Kohn-Sham orbitals and energy eigenvalues. The results show that the random-phase approximation polarizability $\alpha_{R P A}$ divided by the volume of the molecules decreases with increasing $n$. This behavior is in contrast to the indications of the spherical model proposed in the paper by Knize [4], as well as those of our present 2D jellium model, and seems to suggest strong localization of the electrons about each of the $\mathrm{C}$ nuclei on the surface of the molecule.

It is worth noting here that high-order-harmonic generation (HHG) has been investigated in $\mathrm{C}_{60}$. The HHG spectrum of $\mathrm{C}_{60}$ has been predicted using a theoretical model where the valence electrons are contained inside a spherical shell of finite thickness [31] and also by a model where a single electron is constrained over the surface of a rigid sphere [32]. The spectrum consists of odd harmonic peaks of the driving laser frequency, in addition to hyper-Raman lines. Experimentally, the $\mathrm{C}_{60}$ spectrum has been obtained in a plasma plume [33-36].

\section{COMMENTS}

In this section we shall compare the physical properties predicted by our toy model in Sec. II with those summarized in Sec. III for hollow clusters. Given the simplicity of our model, we shall focus on qualitative trends rather than on quantitative predictions.

It is easy to check that the number of magic members between 0 and $M$ is obtained from Eq. (4) as

$$
n(M)=\sqrt{\frac{M}{2}}-1
$$

The density of magic numbers is

$$
\rho(M) \equiv \frac{d n(M)}{d M}=\frac{M^{-1 / 2}}{2 \sqrt{2}},
$$

which shows that the density of magic numbers decreases according to a rather mild power-law distribution. Random and infrequent processes, such as the number of tsunami

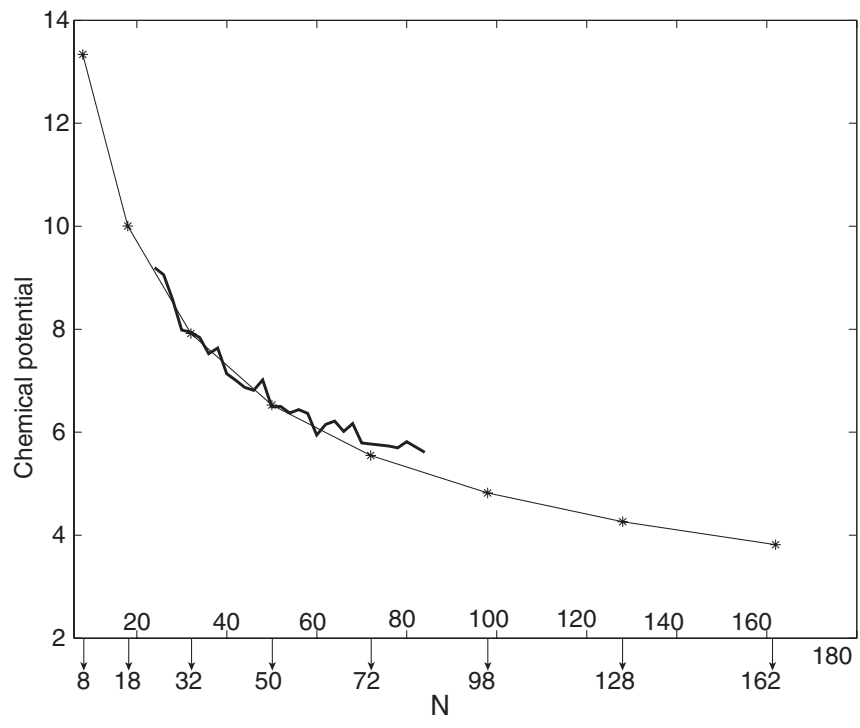

FIG. 9. Starred line: the stability polygonal in eV from Fig. 3 of the present paper. Thick line: binding energy for different fullerenes. Data are adapted from Fig. 1 of [25].

occurring in 1 year, obey the Poisson distribution and vanish in a quasiexponentially fast fashion. Square-root power-law distributions are rather common in statistics and have been found to describe a large variety of cases such as wealth in a
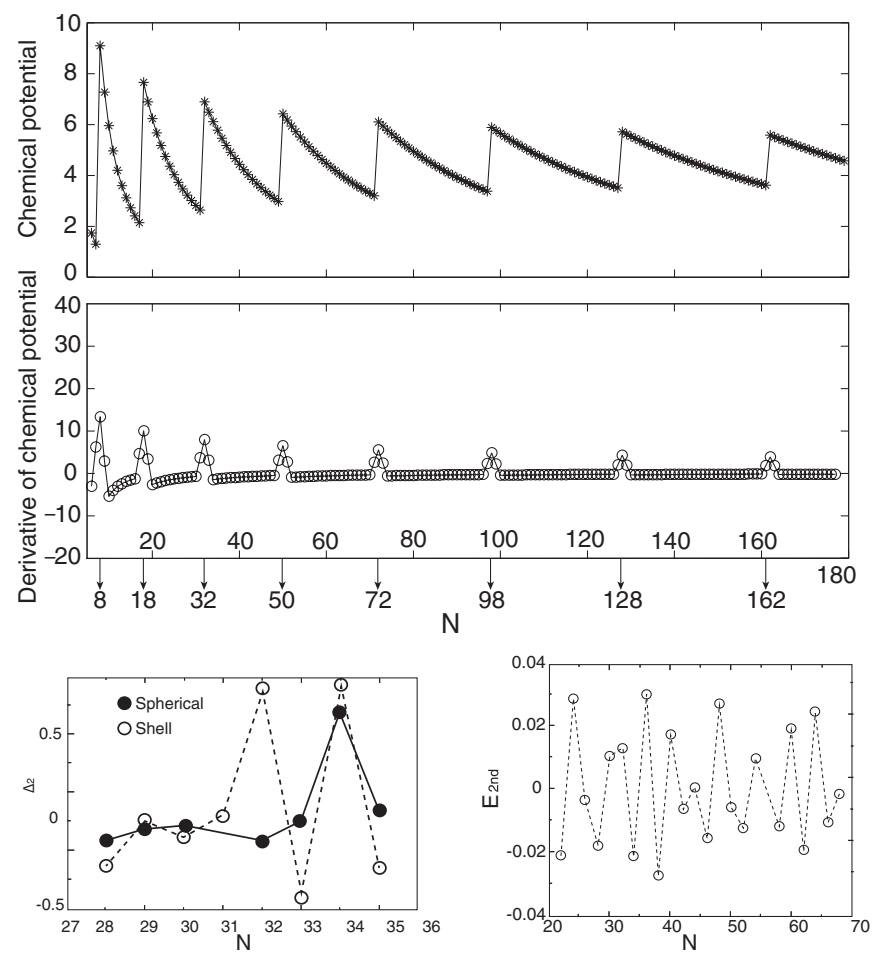

FIG. 10. (top) The chemical potential $\mu(N) \equiv W_{N, N}-$ $W_{N-1, N-1}$ of the system in $\mathrm{eV}$ vs the number $N$ of electrons (the solid line is an interpolation) and (middle) second derivative of $W_{N, N}$; both plots are from Fig. 3 of the present paper. (bottom left) Stability peaks of the $\mathrm{Mg}^{+} \mathrm{O}^{-}$clusters; data are adapted from Fig. 4 of [16]. (bottom right) Stability peaks for the shell model of the ground states of the clusters reported in Fig. 6 of [26] from which the data are adapted. 

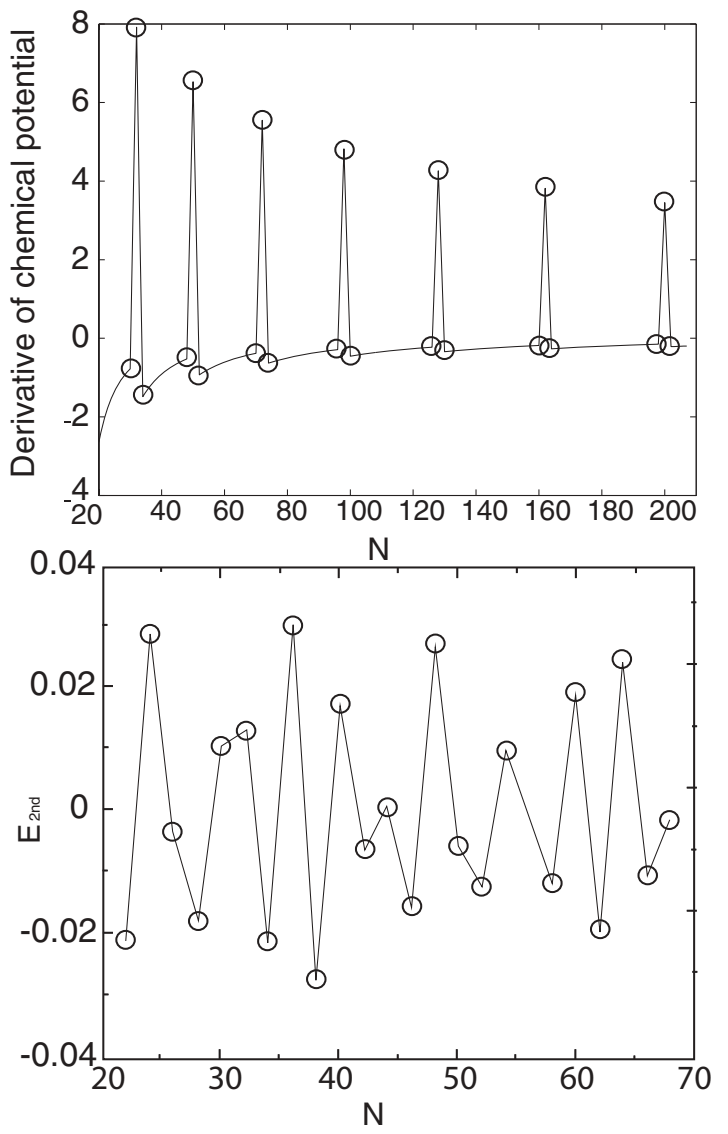

FIG. 11. (top) The second derivative of $W_{N, N}$ in $\mathrm{eV}$ vs the number $N$ of electrons; from Fig. 3 of the present paper. (bottom) Stability peaks points adapted from Fig. 6 of [26].

population or earthquake intensity distribution. Of course the case here studied is not a random process but the outcome of a perfectly deterministic law. The similarity, however, must be noted and kept in mind for further investigations.

Regarding the chemical potential and stability in Fig. 9 we compare the polygon of Fig. 4 of the present paper with Fig. 1 of [25]. We see that our model seems able to capture the main features of the stability curve of the fullerenes. The appearance of the stability peaks in Fig. 3 of the present paper seems similar to that of the stability peaks of the $\mathrm{Mg}^{+} \mathrm{O}^{-}$ clusters in Fig. 6 of [26] as well as to that of the stability peaks for the shell model of the ground states of the clusters reported in Fig. 4 of [16]. We show these similarities in Fig. 10. In Fig. 11 we compare our second derivative of $W_{N, N}$ with the stability peaks of Fig. 6 of [26].

The trend of the ionization energy of fullerenes $\mathrm{C}_{n}$ as a function of $n$ has been calculated using density-functional theory and is reported in Fig. 1 of [29]. In Fig. 12 of the present paper we compare the lower curve of this figure with the results of our model. The qualitative trend seems to be the same apart from the obvious scale differences (Figs. 11 and 12).

We have addressed also the problem of static polarizability; Figs. 6 and 7 of the present paper indicate an essentially parabolic dependence of the polarizability of our model from the number of electrons. This is at variance with the

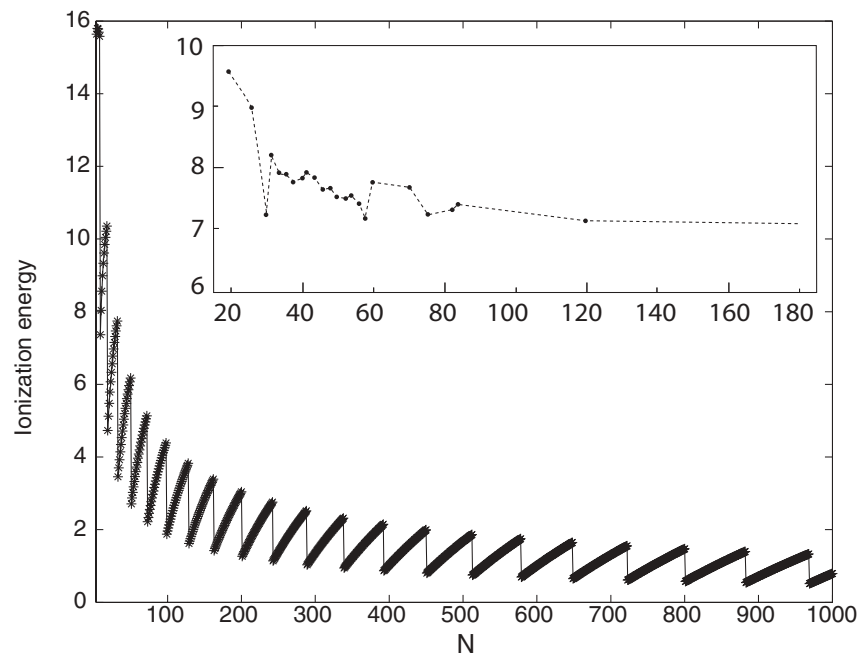

FIG. 12. Ionization potential in $\mathrm{eV}$ vs the number $N$ of atoms extended to 1000 atoms. The inset shows the ionization potential calculated using density functional theory; data are adapted from Fig. 1 of [29].

results of the density-functional theory in the random-phase approximation presented for the extended fullerene series in Fig. 2 of [30]. Evidently, our toy model, consisting of free electrons, is not able to capture the physics of electrons which are strongly localized around the carbon nuclei.

It may be appropriate at this point to spend a few words on the exchange and correlation effect [6]. Using these results, we speculate that exchange and correlation should yield an attractive contribution to the effective electron-electron interaction. Such a contribution, however, is a function of the Seitz radius, which becomes negligible at low electron density.

\section{CONCLUSIONS}

Inspired by John Ziman's ideas on mathematical models in theoretical physics [37] and presumably influenced by many discussions on this subject that took place within his group almost 50 years ago in Bristol and subsequently spread around all the physics world, we have considered a mathematical model consisting of a finite but large number $N$ of noninteracting electrons constrained on a spherical surface. We have calculated a number of physical properties that such an ideal system should possess, with particular reference to chemical stability, ionization potential, electric polarizability, and the generation of high-order harmonics in a strong laser field, and we have compared the results of our calculations with the analogous physical properties experimentally observed (when experimental results are available) or theoretically predicted (by relatively sophisticated theoretical chemistry techniques on much more realistic models) for hollow spherical clusters of atoms and molecules. Given the simplicity of our toy model, we could not expect quantitative agreement. Thus we have focused on trends of the selected physical properties as functions of $N$. Our calculations indicate the prominence of shell effects for all the physical properties considered, yielding a distribution of magic numbers $N$, many of which are observed in hollow quasispherical clusters. All our calculations have been performed analytically, although for reasons of 
clarity the results are summarized numerically in the figures. The trend in the ionization potential for our toy model is qualitatively similar to that obtained for the fullerene series using density-functional theory. In contrast, the electric static polarizability behaves quite differently as a function of $N$ from that obtained using self-consistent density-functional methods; this discrepancy is presumably due to the impossibility for our model to account for effects of localization of the electrons around nuclei of cluster atoms. Finally, it may also be appropriate to remark that the toy model discussed here seems to suggest the possibility of establishing chemical bonds between two different clusters exploiting the formation of closed shells by sharing electrons in incomplete valence shells. By a stretch of imagination one could thus envisage a rich new chemistry of clusters where the building blocks are whole magic clusters rather than atoms and molecules. In this perspective the mathematical model presented here might turn out to be a useful tool for a preliminary design of complex new nanoscale materials involving hollow molecules.

\section{ACKNOWLEDGMENTS}

We wish to thank Marvin H. Mittleman for his reading of the manuscript, his comments, and his suggestions in improving the text and Giuseppe Castiglia for help in debugging computer codes.
[1] M. Koskinen, P. O. Lipas, and M. Manninen, Z. Phys. D 35, 285 (1995).

[2] P.-F. Loos and P. M. W. Gill, Phys. Rev. B 83, 233102 (2011).

[3] N. Mizorogi, M. Kiuchi, K. Tanaka, R. Sekine, and J. Aihara, Chem. Phys. Lett. 378, 598 (2003).

[4] R. J. Knize, Opt. Commun. 106, 95 (1994).

[5] A. Hernando, P. Crespo, and M. A. Garcia, J. Phys. Conf. Ser. 292, 012005 (2011).

[6] P.-F. Loos and P. M. W. Gill, J. Chem. Phys 135, 214111 (2011).

[7] P. M. W. Gill and P.-F. Loos, Theor. Chem. Acc. 131, 1069 (2012).

[8] R. C. T. da Costa, Phys. Rev. A 23, 1982 (1981).

[9] M. Lassaut, R. J. Lombard, and R. Yekken, Phys. Scr. 78, 035008 (2008).

[10] C. Ortix and J. van den Brink, Phys. Rev. B 81, 165419 (2010).

[11] P. C. Schuster and R. L. Jaffe, Ann. Phys. (NY) 307, 132 (2003).

[12] P. Maraner, J. Phys. A 29, 2199 (1996).

[13] R. P. Feynman, Statistical Mechanics: A Set of Lectures (Westview, Boulder, CO, 1998).

[14] A. V. Verkhovtsev, R. G. Polozkov, V. K. Ivanov, and A. V. Solov'yov, Phys. Scr. 80, 048104 (2009).

[15] R. G. Polozkov, V. K. Ivanov, A. V. Verkhovtsev, and A. V. Solov'yov, Phys. Rev. A 79, 063203 (2009).

[16] W. J. Yin, X. Gu, and X. G. Gong, Solid State Comm. 147, 323 (2008).

[17] X. Gu, M. Ji, S. H. Wei, and X. G. Gong, Phys. Rev. B 70, 205401 (2004).

[18] D. Tian, B. Wang, and B. King, J. Phys. Chem. A 111, 411 (2007).

[19] P. W. Fowler and D. E. Manolopoulos, An Atlas of Fullerenes (Dover, Mineola, NY, 2006).

[20] P. R. C. Kent, M. D. Towler, R. J. Needs, and G. Rajagopal, Phys. Rev. B 62, 15394 (2000).
[21] H. Kietzmann, R. Rochow, G. Ganteför, W. Eberhardt, K. Vietze, G. Seifert, and P. W. Fowler, Phys. Rev. Lett. 81, 5378 (1998).

[22] V. J. Kovalenko and A. R. Khamatgalimov, Russ. Chem. Rev. 75, 981 (2006).

[23] L. Xu, W. Cai, and X. Shao, Comput. Mater. Sci. 41, 522 (2008).

[24] P. W. Fowler, T. Heine, and F. Zerbetto, J. Phys. Chem. A 104, 9625 (2000).

[25] A. S. Fedorov, D. A. Fedorov, A. A. Kuzubov, P. V. Avramov, Y. Nishimura, S. Irle, and H. A. Witek, Phys. Rev. Lett. 107, 175506 (2011).

[26] C. Roberts and R. L. Johnston, Phys. Chem. Chem. Phys. 3, 5024 (2001).

[27] O. V. Boltalina, I. N. Ioffe, L. N. Sidorov, G. Seifert, and K. Vietze, J. Am. Chem. Soc. 122, 9745 (2000).

[28] H. Steger, J. Holzapfel, A. Hielscher, W. Kamke, and I. V. Hertel, Chem. Phys. Lett. 234, 455 (1995).

[29] G. Seifert, K. Vietze, and R. Schmidt, J. Phys. B 29, 5183 (1996).

[30] R. R. Zope, T. Baruah, M. R. Pederson, and B. I. Dunlap, Phys. Rev. B 77, 115452 (2008).

[31] M. F. Ciappina, A. Becker, and A. Jaroń-Becker, Phys. Rev. A 76, 063406 (2007).

[32] D. Cricchio, P. P. Corso, E. Fiordilino, G. Orlando, and F. Persico, J. Phys. B 42, 085404 (2009).

[33] R. A. Ganeev, L. B. Elouga Bom, M. C. H. Wong, J.-P. Brichta, V. R. Bhardwaj, P. V. Redkin, and T. Ozaki, Phys. Rev. A 80, 043808 (2009).

[34] R. A. Ganeev, L. B. Elouga Bom, J. Abdul-Hadi, M. C. H. Wong, J. P. Brichta, V. R. Bhardwaj, and T. Ozaki, Phys. Rev. Lett. 102, 013903 (2009).

[35] R. A. Ganeev, H. Singhal, P. A. Naik, J. A. Chakera, A. K. Srivastava, T. S. Dhami, M. P. Joshi, and P. D. Gupta, Appl. Phys. B 100, 581 (2010).

[36] R. A. Ganeev, Laser Phys. 21, 25 (2011).

[37] J. M. Ziman, Nature (London) 206, 1187 (1965). 